\title{
Characterization and Antimicrobial Activity of ECO-Friendly Biosynthesis of Silver Nano Particles Using An Aqueous Leaf Extract of Catharanthus Roseus
}

\author{
S.Senthilkumar, E.Siva and A.Rajendran*, \\ $P G$ and Reseach Department of Physics, Nehru memorial College (Autonomous), Puthanampatti, Trichy, \\ Tamilnadu-621007, India.
}

\begin{abstract}
A green rapid biogenic synthesis of silver nanoparticles (AgNPs) using Catharanthus roseus (C. roseus) aqueous extract. Synthesized nanoparticles were characterization using UV-Visible, FTIR, XRD, FESEM and HRTEM. The reductions of silver ions to AgNPs using C. roseus extract were completed within 10 mins. The formations of AgNPs were confirmed by using Surface Plasmon Resonance (SPR) at $413 \mathrm{~nm}$ using $U V$-Vis spectrophotometer. From the morphological studies, revealed the spherical shape of the particles with sizes ranging from $45 \mathrm{~nm}$ and the EDAX spectrum confirmed the presence of silver along with other elements in the plants metabolite. The extracellular AgNPs synthesis by aqueous leaf extract demonstrates ultra-fast, simple and inexpensive method comparable to other methods. The colloidal AgNPs were found to exhibit antibacterial activity against Enterobacter aerogenes, Proteus vulgaris and Eggerthella sp. Since these compounds are also safe to use and discharged into the environment, the green-AgNPs could be considered as an innovative alternative approach for biomedical and nanoscience based industrials.
\end{abstract}

Keywords: Antibacterial activity, Catharanthus roseus, Eco-friendly, Green synthesis, AgNPs

\section{Introduction}

Nanotechnology is anticipated to open novel opportunities to prevent and fight against diseases using atomic scale tailoring of materials [1]. In the field of nanotechnology, the synthesis of nanoparticles of different sizes, chemical compositions, and controlled monodispersity is essential because they exhibit unique properties, which are not observed in bulk materials [2-3]. Nanoparticles play crucial roles in drug delivery, diagnostics, imaging, sensing, gene delivery, artificial implants and tissue engineering [4] and which has considerable attention because of their various applications. The novel green synthesis of silver nanoparticles (AgNPs) is evolving an important branch of nanotechnology. Recently, researchers have focused on biologically synthesized AgNPs, because of its extensive applications in the development of new technology in the field of electronics, material science and medicine at the nanoscale [5]. Catharanthus roseus (L.) G. Don (Vinca rosea) (Local Name in Tamil: Nithyakalyani), an important medicinal plant, belongs to family Apocynaceae and it is a perennial herb grown commercially for its alkaloids [6-7]. There are more than 70 alkaloids (mostly of indole type) that have been reported from different parts of C. roseus [8] and the major alkaloids are Vincristine and Vinblastine, which has anticancer property and used as a drug in the treatment of different cancers [9]. In India as well as other countries, tea made from the fresh leaf juice of C.roseus has been used by Ayurvedic physicians for the treatment of certain skin problems such as dermatitis, eczema and acne [10]. AgNPs also show evidence of potent cytoprotective activity towards HIV-infected cells [11]. The main aim of this study is to green synthesize AgNPs by reducing silver ions present in the fresh aqueous leaf extract of the herbal plant C.roseus and analyzeits antibacterial activity against gram negative and gram positive bacteria.

Plant Collection and Biosynthesis of Silver Nanoparticles

\section{Experimental}

The fresh, fully matured leaves of $C$. roseus were collected from Bharathidasan University campus Tiruchirappalli, India and leaves were cut and shade dried which is used for the biosynthesis of AgNPs. Plant leaf extracts were prepared by boiling $10 \mathrm{~g}$ of dried leaves in $90 \mathrm{ml}$ of deionized water for 30 minutes at $60^{\circ} \mathrm{C}$ and filtered through a Whatmann no.1 filter paper. C. roseus leaf extracts was treated with 90 Mole aqueous $1 \mathrm{mM} \mathrm{AgNO}$ solution with constant stirring for reduction of $\mathrm{Ag}+$ ions and then periodically $(10,30,60,120$ and 180 mins) color change was observed.

National Conference on Current Advancements in Physics $3^{\text {rd }} \& 4^{\text {th }}$ February 2017 


\section{Characterizations of the synthesized Silver nanoparticles}

The pure synthesized AgNPs in presence of the leaf extract was monitored by observing the UV-Vis (UV-2450, Shimadzu, and Kyoto, Japan) analysis at 300-600 nm. The biosynthesized AgNPs solutions were analysis by FTIR spectroscopy in the range of 400 to $4000 \mathrm{~cm}^{-1}$ to identify its functional groups. The XRD pattern was recorded using $\mathrm{CuK} \alpha$ radiation $\left(\lambda=1.54060 \mathrm{~A}^{\circ}\right)$ with copper monochromator in the range of $2 \Theta$ from $10^{\circ}$ to $80^{\circ}$. The morphological features of synthesized AgNPs from C. roseus extract were studied by Field Emission Scanning Electron Microscopy (FESEM). The elemental composition of the synthesized nanoparticles was analyzed with Energy Dispersive spectroscopy coupled to scanning electron microscope (JEOL - Model 6390).

\section{Antimicrobial Activity}

The antimicrobial activity of AgNPs was estimated against the pathogenic activity of two Gram negative (E. aerogenes and P. vulgaris) and one Gram positive (Eggerthella sp) bacteria using a disk diffusion method. The three concentrations $(100 \mu \mathrm{l}, 200 \mu 1$ and $300 \mu \mathrm{l} / \mathrm{ml}$ ) of synthesized AgNPs in DMSO (negative control), were loaded onto sterile discs with a diameter of $6 \mathrm{~mm}$, and placed in the respective plates. After $24 \mathrm{hrs}$ incubation at $37^{\circ} \mathrm{C}$, the zone of inhibition was measured and the strains classified based on their susceptible activity [12].

\section{Results and Discussion}

\section{Biosynthesis of Silver Nanoparticles and UV-Vis Spectral Analysis}

In the emerging field of nanotechnology, the formation of nanoparticles using biological materials as reducing agents is receiving more attention, especially in the last decade [13]. Successful biosynthesis of AgNPs by the filtrate of $C$. roseus was confirmed by the change in the color of the filtrate from yellow to dark brown after the addition of $\mathrm{AgNO}_{3}$ but control flasks, containing only the $\mathrm{AgNO}_{3}$ solution (without plant filtrates) did not show any color change. AgNPs have free electrons which have risen to SPR absorption due to the vibration of electrons of the metal nanoparticles in resonance with light wave and it is an indication of formation of AgNPs [14]. The green synthesized nanoparticles at different time interval (10, 30, 60, 120 and 180 mins) was analyzed UV-Vis spectroscopy at $300-600 \mathrm{~nm}$ and the UV-Visible spectrum of AgNPs was recorded and the formation of AgNPs was confirmed by silver SPR peak ranging 413-424nm (Fig.1A).

Further the result of the UV-Vis spectra of the biosynthesized AgNPs were confirmed by the characteristic peaks observed in the XRD image and the structural view under the scanning electron microscope as shown in Figure.1B. In this result, the XRD pattern showed intense peaks at $38.07^{\circ}, 44.26^{\circ}, 64.41^{\circ}, 77.36^{\circ}$ which can be index the angles values of (111), (200), (220) and (240), respectively for crystalline planes of nano silver. The average of grain size of the AgNPs formed it was estimated at $32 \mathrm{~nm}$. The calculated lattice constant from this pattern was $4.085 \mathrm{~A}^{\circ}$. The XRD results clearly show that AgNPs was formed by the reduction $\mathrm{Ag}+$ ions AgNPs and revealed the spherical structure of AgNPs and the high peaks indicates the active silver composition and crystalline nature of the AgNPs. The compound is stable, which is confirmed by its angle value. The possible interactions of AgNPs with different functional groups and efficient stabilization were analysed using FTIR analysis and the resulting spectrum shows the presence of different functional groups at various positions (Fig.1C) which also confirmed that the bio reduction of silver ions to AgNPs by capping material of plant extract. The spectra had maximum peaks at $885.16 \mathrm{~cm}^{-1}, 3002.99 \mathrm{~cm}^{-1}$, indicating the presence $\mathrm{C}-\mathrm{O}$ stretch bond, $\mathrm{C}=\mathrm{C}$ group. The overall observation confirms that AgNPs obtained through green synthesis were more stable.
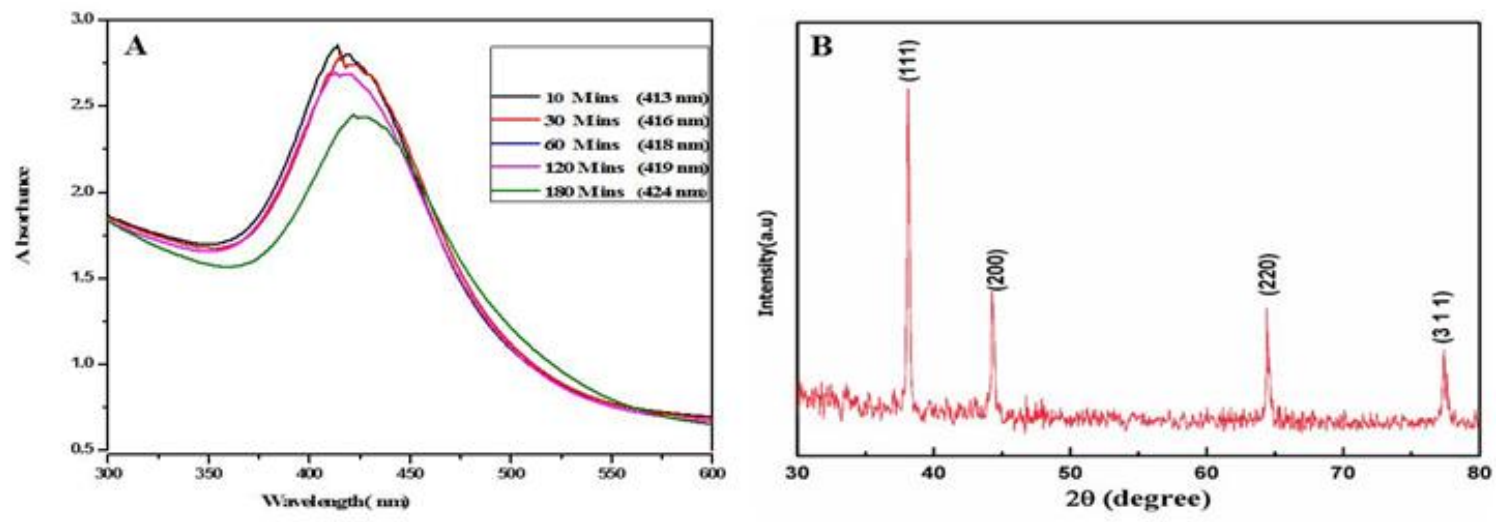

National Conference on Current Advancements in Physics $3^{\text {rd }} \& 4^{\text {th }}$ February 2017 


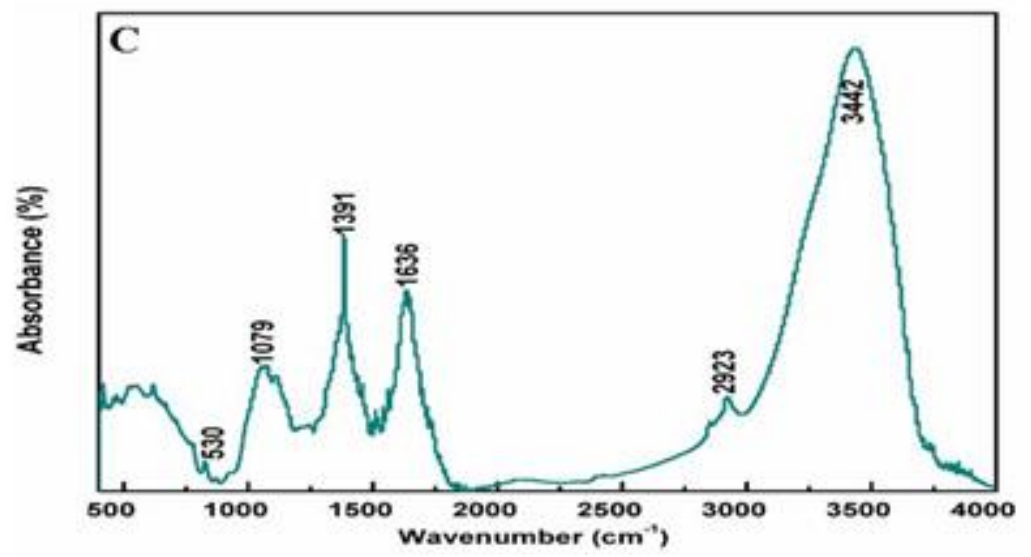

Fig. 1 (A) UV-Vis absorption spectra of synthesized AgNPs with different time Intervals; (B) XRD patterns of AgNPs by treating with $\mathrm{AgNO}_{3}$ solution; (C) FTIR spectra of AgNPs.

Figure.2A and $\mathrm{C}$ shows the morphology of AgNPs investigated using FESEM. It reveals that the green synthesized AgNPs particles are spherical in shape with agglomerations, and the dimensions of the spherical shape are between $45 \mathrm{~nm}$ in width and smooth surface of AgNPs are well dispersed. The EDAX revealed the higher percentages of silver peaks which confirm the presence of elemental silver, which indicates bio-reduction of silver ion to elemental silver (AgNPs) and peaks for chlorine were also observed (Fig.2D). The strong signal in the silver region was observed at $4 \mathrm{keV}$ for AgNPs due to the SPR [15].

The morphology and internal crystalline structure of obtained nanoparticles was studied by the High Resolution transmission electron microscopy (HRTEM and SAED) and patterns were observed as shown in Figure. 2E and F. The HRTEM microscopy revealed that a spherical shape and a grain like morphology of AgNPs (Fig.2E). The HRTEM micrograph of the synthesized AgNPs is at $200 \mathrm{~nm}$ scale. The selected area electron diffraction (SAED) patterns confirmed the presence of elemental AgNPs as shown in Figure. 2F. The XRD, FESEM and HRTEM analyses showed the particle size between $48 \mathrm{~nm}$ as well the spherical structure of the nanoparticles. Similarly, the phenomenon was reported by Chandran et al., in 2006 [16] and Mukuthan et al., in 2011 [17].
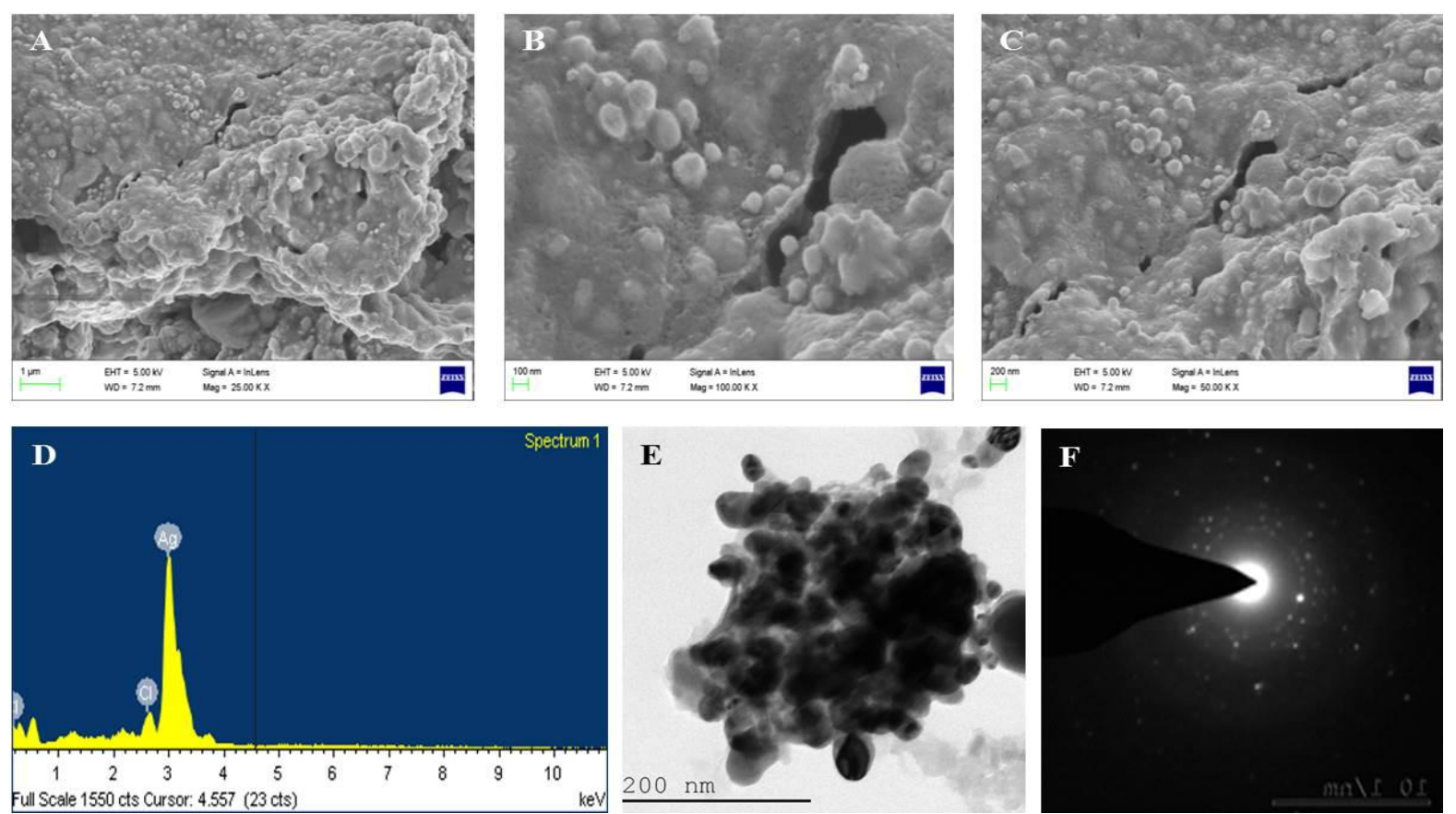

Fig.2 (A-C) FESEM image of AgNPs at different magnification; (D) EDAX spectrum of AgNPs; (E) HRTEM images of synthesized AgNPs; (F) HRTEM image SAED patterns ofAgNPs. 

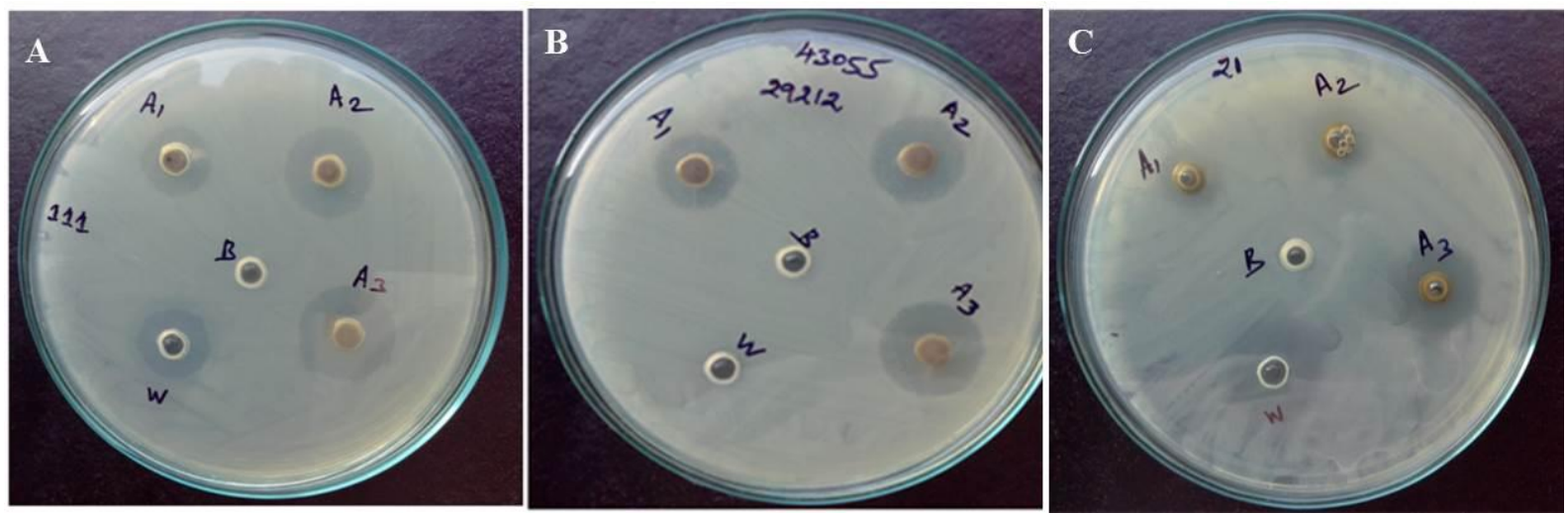

Fig.3 Antimicrobial activity of synthesizedAgNPson; (A) Enterobactor aerogenes(B) Proteous Vulgaris and

(C) Eggerthella sp by the disk diffusion method.

Table 1 Zone of inhibition produced by Gram negative and Gram positive against synthesized AgNPs.

\begin{tabular}{|l|l|l|l|l|}
\hline S.No & Volume & E. aerogenes & P. vulgaris & Eggerthella $\mathbf{s p}$ \\
\hline $\mathbf{1}$ & $100 \mu \mathrm{l}$ & $10 \mathrm{~mm}$ & $13 \mathrm{~mm}$ & $17 \mathrm{~mm}$ \\
\hline $\mathbf{2}$ & $200 \mu \mathrm{l}$ & $17 \mathrm{~mm}$ & $15 \mathrm{~mm}$ & $14 \mathrm{~mm}$ \\
\hline $\mathbf{3}$ & $300 \mu \mathrm{l}$ & $19 \mathrm{~mm}$ & $17 \mathrm{~mm}$ & $16 \mathrm{~mm}$ \\
\hline
\end{tabular}

The antibacterial activities of solutions containing different concentrations of AgNPs were showed in

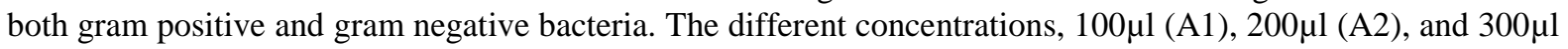
(A3) of AgNPs was used for antimicrobial activity assay, which disturbs the surface of the bacterial cell membrane therefore disturbing the permeability and respiration functions. The maximum antibacterial activity in $300 \mu \mathrm{l} / \mathrm{ml}$ concentration of the synthesized AgNPs was $19 \mathrm{~mm}, 17 \mathrm{~mm}$ and $16 \mathrm{~mm}$ for E. aerogenes, $P$. vulgaris and Eggerthella sp, receptively. E. aerogenes shows highest antibacterial activity for synthesized AgNPs by $C$. roseus leaf extract than other bacteria. This confirms that AgNPs from C. roseus exhibited good antibacterial potential against gram-negative bacterial strains when compared to gram positive strain. The result shows that the zone of inhibition (ZOI) was increased when increasing the concentration of AgNPs. The results of the antibacterial assay are depicted in Figure. 3 and Table 1. These results confirm previous work carried out by Li et al. 2011 [18] and Bindhu and Uma Devi (2013) [19]. Similarly, Ponarulselvam et al. (2012) [8] and Kotakadi et al. (2013) has reported green synthesis of AgNPs from C. roseus leaf extract and the nanoparticles with an average size of 30-55 $\mathrm{nm}$. They have reported that proven active against malaria parasite Plasmodium falciparum and P. fluorescens, at the lowest concentration of the AgNPs $[8,20]$.

The application result indicated and confirmed that AgNPs have good antimicrobial activity against different microorganisms and hence it could have great impact on eco-friendly mode of drug treatment for bacterial diseases. More than that, this method could be useful for different field of science like medicine and drug delivery in a safer way.

\section{Conclusion}

In conclusion, the leaf extract of $C$. roseus are excellent source for synthesizing AgNPs. It is an ecofriendly, cost effective, method which is very simple to produce. This study also reveals that synthesized AgNps show antibacterial activity in both gram positive and gram negative bacteria, but is more effective for gram negative organisms when compared with gram positive organisms, because of its significant antibacterial efficacy, it may have potential applications in the field of biomedicine. Finally, it can be extendable to large scale production of AgNPs for commercial use.

\section{References}

[1] 1. R. Bhuvaneswari, R. John Xavier and M. Arumugam (2015). IOSR Journal of Applied Physics. 7, 81.

[2] P.T. Lakshmi, D. Priyanka and A. Annamalai (2015). Int. J. Biomater. 2015, 539494.

[3] I. Moreno-Garrido, S. Perez and J. Blasco J (2015). Mar. Environ. Res. 111, 73.

[4] J.R. Morones, J.L. Elechiguerra, A. Camacho, K. Holt, J.B. Kouri and J.T. Ramirez (2005). Nanotechnology. 16, 2353.

[5] L. Xia, S.C. Lenaghan, M. Zhang, Z. Zhang and Q. Li (2010). J. Nanobiotechnology. 8, 9.

[6] S. Hossain, M. Hossain, Z. Haque and P.K. Moyen Uddin (2015). Int. J. Bioassays. 4,610.

[7] J. Kandhavelu, N. Krishnan, M. Kandhavelu (2012). Immunopharmacol Immunotoxicol. 34, 281.

[8] S. Ponarulselvam, C. Panneerselvam, K. Murugan, N. Aarthi, K. Kalimuthu and S. Thangamani (2012). Asian Pac. J. Trop. Biomed. 2, 580. 
[9] P.K. Fischhof, R. Moslinger-Gehmayr, W.M. Herrmann, A. Friedmann and D.L. Russmann (1996). Neuropsychobiology. 34, 35 .

[10] K. Syam Sree, M. Anudeep, C.H. Venkata Ramana and C.H. Bhaskar (2015). J. Pharmaco. Gn. Phytochemical. 3, 156.

[11] R.W. Sun, R. Chen, N.P. Chung, C.M. Ho, C.L. Lin and C.M. Che (2005). Chem. Commun (Camb). 40, 5061.

[12] P. Prakash, P. Gnanaprakasam, R. Emmanuel, S. Arokiyaraj and M. Saravanan (2013). Colloids Surf. B. Biointerfaces. 108, 259.

[13] S.M. Roopan, T.V. Surendra, G. Elango and S.H. Kumar (2014). Appl. Microbiol. Biotechnol. 98,5300.

[14] M. Villanueva-Ibáñez, M.G. Yañez-Cruz, R. Álvarez-García, M.A. Hernández-Pérez and M.A. Flores-González (2015). Materials Letters.152, 169.

[15] S. Kaviya, J. Santhanalakshmi, B. Viswanathan, J. Muthumar and K. Srinivasan (2011). Spectrochimica. Acta. Part A: Molecular and Biomolecular Spectroscopy. 79, 598.

[16] S.P. Chandran, M. Chaudhary, R. Pasricha, A. Ahmad and M. Sastry (2006). Biotechnol Prog. $22,583$.

[17] K.S. Mukunthan, E.K. Elumalai, T.N. Patel and V.R. Murty (2011). Asian. Pac. J. Trop. Biomed.1, 274

[18] W.R. Li, X.B. Xie, Q.S. Shi, S.S. Duan, Y.S. Ouyang and Y.B. Chen (2011). Biometals. $24,141$.

[19] M. R. Bindhu and M. Umadevi (2013). Spectrochim Acta. A. Mol. Biomol. Spectrosc. 101,190.

[20] V.S. Kotakadi, Y.S. Rao, S.A. Gaddam, T. N. Prasad, A.V. Reddy and D.V Gopal 2013). Colloids Surf. B. Biointerfaces.105, 198. 